\title{
ON THE FRIEDMANN COSMOLOGY
}

\author{
Dismas S. Wamalwa \\ Department of Physics \\ University of Nairobi Chiromo, Nairobi, 30197-00100, KENYA
}

\begin{abstract}
We derive a functional relation between redshift and light intensity assuming an underlying Friedmann-Robertson-Walker metric without invoking the effects of dark matter and dark energy. Our relation, which is applicable to direct observational data that does not involve distance measurements, is consistent with classical observations for nearby stars and galaxies.
\end{abstract}

AMS Subject Classification: 83,85

Key Words: redshift, light intensity, Robertson-Walker metric, isotropic, homogeneous

\section{Introduction}

For a long time the distribution of galaxies in the universe is believed to be homogeneous and isotropic on large scale. The experimental evidence for this belief was taken from the distribution of galaxies in the sky; the linearity of the redshift-distance relation (Hubble law); the isotropic distribution of the radio sources in the sky and the isotropy of the cosmic micro-wave background radiation (CMBR) [1] and [2].

However, these facts together with the cosmological principle which says that the universe is homogeneous and isotropic have faced challenges in recent years [1]. This is because theoretical cosmology employs physical laws established on the earth, and makes the daring extrapolation that they apply throughout the universe. But Physics even when extrapolated is not enough [1]. This is because the situation about the universe may not look the same

Received: October 1, 2015

Published: May 4, 2016 (c) 2016 Academic Publications, Ltd.

url: www.acadpubl.eu 
for observers not located on the earth. To escape from the prison of the single vantage point, there was therefore need for something more: a cosmological principle. The principle is essentially philosophical in nature, and seems to complement the laws of Physics rather than follow from them.

Currently, big catalogues have been compiled that list objects in the sky and give their distance from us as well as the direction from which their light reaches us. We thus, obtain a three-dimensional picture of the luminous matter in the universe. While the two-dimensional projection that we see by looking at the sky seems to be fairly isotropic, the three-dimensional distribution seems to show inhomogeneity at almost all scales [3], [4], and [5]. This is philosophically disturbing: the most natural and naive expectation, confirmed by the isotropy of the two-dimensional projection, was to assume that the universe looks isotropic to us. Then the cosmological principle says that it must look the same to every observer in the universe (at rest with respect to the background) [1], [6] and [7]. So the observations showing that the three-dimensional distribution of galaxies seem to show inhomogeneities at all scales came as a big surprise.

The three-dimensional maps of the universe depicting inhomogeneities depend on our ability to measure distance. The distance measurements involve a complicated ladder of measurements proceeding from small distances to ever larger distances. At each rung of this ladder, some physical assumptions have to be made [1], [8] and [9]. In more or less hidden form, the assumption that the universe is Friedmann may enter into these measurements. Yet these same catalogues seem to raise the question as to whether the universe is indeed Friedmann.

IAU symbosium 289 addressed the physics underlying methods of distance determination across the universe, exploring the various approaches involved and acknowledging that the controversy has, thus, not been solved, and all methods applied to date are affected by their own unique sets of uncertainties [9]. So, methodologically, it would be clearer to try to address that latter question using direct observations which do not involve assumptions about the space-time.

Sloan Digital Sky Survey (SDSS) which constructs the largest map of the universe only maps about a quarter of the sky in three-dimension. However, this volume-limited latest SDSS data reveal clustering of galaxies that point towards inhomogeneities even to largest scales [10] and [11]. Therefore, only a bigger map could resolve the current dispute associated with volume-limited statistical data. Although the technology is not yet able to probe wider and deeper into far off astronomical objects to provide us with huge direct observations, we are 
optimistic we shall soon be there with the current advances. In addressing this problem, we thus, assume that we are given huge and direct observational data that does not involve distance measurements. From such data, we investigate if it is possible to rule out that luminous matter is homogeneously distributed in accordance with the Friedmann universe.

In trying to answer the question of how much we can learn from astronomical observations that involve no assumptions about the background geometry, we shall consider two astronomical quantities that can easily be measured: the redshift $z$ of light from a given astronomical object and the light intensity $z$ from such an object. Suppose we are given a large list of all astronomical objects for which the above quantities have been measured together with the direction from which the light comes. A naturally arising question is whether such data is compatible with the assumption that the universe is described by the Friedmann metric. To satisfactorily answer this question, one needs to investigate the functional dependencies of these two quantities assuming an underlying Friedmann metric, and then prepare the results for comparison with observed dependencies. In this paper, we lay the basis for answering this question by developing a functional relationship between light intensity $I$ and redshift $z$ without invoking the presence of dark matter and dark energy. The established relationship shall form the basis of our future work in preparing the results for comparison with future observed dependencies.

\section{Review of Friedmann Model}

One of the most widely accepted cosmological models is the Friedmann model [6] and [10]. The model describes an isotropic and dynamic universe. In this model, the cosmological principle is implemented as follows: there is a preferred time coordinate $t$ such that the $t=$ constant slices are homogeneous and isotropic spaces. There are basically three simply connected isotropic and homogeneous spaces: the sphere (constant positive curvature), flat space (no curvature), and hyperbolic space (constant negative curvature). By means of stereographic projection, the three cases can be handled in a uniform manner. The metric of the Friedmann universe then reads:

$$
g=c^{2} d t^{2}-\frac{R(t)^{2}}{\left(1+\kappa r^{2}\right)^{2}}\left(d x^{2}+d y^{2}+d z^{2}\right)
$$

where $r^{2}=x^{2}+y^{2}+z^{2}$ (in rectangular coordinate system on the threedimensional projection space); $\kappa=1,0,-1$ for the spherical, flat, hyperbolic 
cases, respectively; and $R(t)$ is the cosmic scale factor (the radius of the universe at time $t$ for the case of the unit sphere $\kappa=1)$; while $c$ is the speed of light.

Since we have assumed an underlying Friedmann metric, we need Einstein's field equations describing the dynamic and evolution of the universe based on this metric.

\subsection{Einstein's Field Equations based on the Friedmann Metric}

To derive Einstein's field equations describing the dynamics and evolution of the universe based on the Friedmann metric, we need the $\Gamma$-symbols of the Friedmann metric. An efficient way of computing them is to consider the functional:

$$
l=\int_{\tau_{0}}^{\tau_{1}} c^{2} \dot{t}^{2}-\frac{R^{2}(t)}{\left(1+\kappa r^{2}\right)^{2}}\left(\dot{x}^{2}+\dot{y}^{2}+\dot{z}^{2}\right) d \tau
$$

where $r^{2}=x^{2}+y^{2}+z^{2}$ and the dot above the time or space coordinate denotes differentiation of that coordinate w.r.t the parametrization $\tau$. The $\Gamma$-symbols can be read off from the coefficients of the quadratic terms in the dotted coordinates in the Euler-Lagrange equations:

$$
\begin{aligned}
& \frac{d}{d \tau}\left(\frac{\partial l}{\partial \dot{t}}\right)=\frac{\partial l}{\partial t} \\
& \frac{d}{d \tau}\left(\frac{\partial l}{\partial \dot{x}}\right)=\frac{\partial l}{\partial x} \\
& \frac{d}{d \tau}\left(\frac{\partial l}{\partial \dot{y}}\right)=\frac{\partial l}{\partial y}
\end{aligned}
$$

and

$$
\frac{d}{d \tau}\left(\frac{\partial l}{\partial \dot{z}}\right)=\frac{\partial l}{\partial z}
$$

Calculating the $\Gamma$-symbols that can be obtained from equation (3), we have:

$$
\frac{d}{d \tau}\left(2 c^{2} \dot{t}\right)=\frac{-2 R(t) R^{\prime}(t)}{\left(1+\kappa r^{2}\right)^{2}}\left(\dot{x}^{2}+\dot{y}^{2}+\dot{z}^{2}\right)
$$

where

$$
R^{\prime}(t)=\frac{d R}{d t}
$$


Equation (7) reduces to the form:

$$
\ddot{t}+\frac{R(t) R^{\prime}(t)}{c^{2}\left(1+\kappa r^{2}\right)^{2}}\left(\dot{x}^{2}+\dot{y}^{2}+\dot{z}^{2}\right)=0 ;
$$

which is the $t$-component of the geodesic equation:

$$
\ddot{x}^{\mu}+\Gamma_{\rho \sigma}^{\mu} \dot{x}^{\rho} \dot{x}^{\sigma}=0
$$

The non-vanishing $\Gamma$-symbols of the form $\Gamma^{0}{ }_{\rho \sigma}$ are therefore:

$$
\Gamma_{11}^{0}=\Gamma_{22}^{0}=\Gamma_{33}^{0}=\frac{R(t) R^{\prime}(t)}{c^{2}\left(1+\kappa r^{2}\right)^{2}} .
$$

Similarly, the non-vanishing $\Gamma$-symbols obtained from the remaining EulerLagrange equations can be shown to be:

$$
\begin{gathered}
\Gamma_{10}^{1}=\Gamma_{01}^{1}=\Gamma_{20}^{2}=\Gamma_{02}^{2}=\Gamma_{30}^{3}=\Gamma_{03}^{3}=\frac{R^{\prime}(t)}{R(t)} \\
\Gamma_{11}^{1}=\Gamma_{21}^{2}=\Gamma_{12}^{2}=\Gamma_{13}^{3}=\frac{-2 \kappa x}{1+\kappa r^{2}} \\
\Gamma_{12}^{1}=\Gamma_{21}^{1}=\Gamma_{22}^{2}=\Gamma_{32}^{3}=\Gamma_{23}^{3}=\frac{-2 \kappa y}{1+\kappa r^{2}} \\
\Gamma_{13}^{1}=\Gamma_{31}^{1}=\Gamma_{23}^{2}=\Gamma_{32}^{2}=\Gamma_{33}^{3}=\frac{-2 \kappa z}{1+\kappa r^{2}} \\
\Gamma_{22}^{1}=\Gamma_{33}^{1}=\frac{2 \kappa x}{1+\kappa r^{2}} \\
\Gamma_{11}^{2}=\Gamma_{33}^{2}=\frac{2 \kappa y}{1+\kappa r^{2}} \\
\Gamma_{11}^{3}=\Gamma_{22}^{3}=\frac{2 \kappa z}{1+\kappa r^{2}}
\end{gathered}
$$

To obtain Einstein field equations, we first need to calculate the Ricci tensor $R_{\alpha \beta}$ defined as

$$
R_{\alpha \beta}=R_{\alpha \mu \beta}^{\mu}
$$

where $R^{\mu}{ }_{\alpha \nu \beta}$ are the components of the Riemann tensor. By definition of the Riemann curvature, we know that

$$
R(X, Y, Z)=\nabla_{X}\left(\nabla_{Y} Z\right)-\nabla_{Y}\left(\nabla_{X} Z\right)-\nabla_{[X, Y]} Z
$$


or, expressed in terms of components,

$$
\begin{array}{r}
R_{\nu \rho \sigma}^{\mu} \frac{\partial}{\partial x^{\mu}}=R\left(\frac{\partial}{\partial x^{\rho}}, \frac{\partial}{\partial x^{\sigma}}, \frac{\partial}{\partial x^{\nu}}\right) \\
=\nabla_{\frac{\partial}{\partial x^{\rho}}}\left(\nabla_{\frac{\partial}{\partial x^{\sigma}}} \frac{\partial}{\partial x^{\nu}}\right)-\nabla_{\frac{\partial}{\partial x^{\sigma}}}\left(\nabla_{\frac{\partial}{\partial x^{\rho}}} \frac{\partial}{\partial x^{\nu}}\right)- \\
\nabla_{\left[\frac{\partial}{\partial x^{\rho}}, \frac{\partial}{\partial x^{\sigma}}\right]} \frac{\partial}{\partial x^{\nu}}
\end{array}
$$

The last term on the r.h.s of equation (21) is zero since it is the Lie bracket of coordinate vector fields. Applying the definition

$$
\nabla_{\frac{\partial}{\partial x^{\alpha}}} \frac{\partial}{\partial x^{\beta}}=\Gamma_{\alpha \beta}^{\rho} \frac{\partial}{\partial x^{\rho}}
$$

to equation (21), we get

$$
\begin{array}{r}
R_{\nu \rho \sigma}^{\mu} \frac{\partial}{\partial x^{\mu}}=\nabla_{\frac{\partial}{\partial x^{\rho}}}\left[\Gamma_{\sigma \nu}^{\alpha} \frac{\partial}{\partial x^{\alpha}}\right]-\nabla_{\frac{\partial}{\partial x^{\sigma}}}\left[\Gamma_{\rho \nu}^{\alpha} \frac{\partial}{\partial x^{\alpha}}\right] \\
=\left(\frac{\partial}{\partial x^{\rho}} \Gamma_{\sigma \nu}^{\alpha}\right) \frac{\partial}{\partial x^{\alpha}}+\Gamma_{\sigma \nu}^{\alpha} \Gamma_{\rho \alpha}^{\beta} \frac{\partial}{\partial x^{\beta}}- \\
\left(\frac{\partial}{\partial x^{\sigma}} \Gamma_{\rho \nu}^{\alpha}\right) \frac{\partial}{\partial x^{\alpha}}-\Gamma_{\rho \nu}^{\alpha} \Gamma_{\sigma \alpha}^{\beta} \frac{\partial}{\partial x^{\beta}}
\end{array}
$$

Replacing $\alpha$ by $\mu$ in the first and third terms, $\beta$ by $\mu$ in the second and last terms on the r.h.s of equation (23), yields:

$$
\begin{aligned}
R_{\nu \rho \sigma}^{\mu} \frac{\partial}{\partial x^{\mu}}=\left(\frac{\partial}{\partial x^{\rho}} \Gamma_{\sigma \nu}^{\mu}\right) \frac{\partial}{\partial x^{\mu}}+\Gamma_{\sigma \nu}^{\alpha} \Gamma_{\rho \alpha}^{\mu} \frac{\partial}{\partial x^{\mu}}- & \\
& \left(\frac{\partial}{\partial x^{\sigma}} \Gamma_{\rho \nu}^{\mu}\right) \frac{\partial}{\partial x^{\mu}}-\Gamma_{\rho \nu}^{\alpha} \Gamma_{\sigma \alpha}^{\mu} \frac{\partial}{\partial x^{\mu}}
\end{aligned}
$$

Reading off $R^{\mu}{ }_{\nu \rho \sigma}$ from equation(24) and replacing $\rho$ by $\mu$, we get:

$$
R_{\nu \mu \sigma}^{\mu}=R_{\nu \sigma}=\frac{\partial}{\partial x^{\mu}} \Gamma_{\sigma \nu}^{\mu}+\Gamma_{\sigma \nu}^{\mu} \Gamma_{\mu \alpha}^{\alpha}-\frac{\partial}{\partial x^{\sigma}} \Gamma_{\mu \nu}^{\mu}-\Gamma_{\mu \nu}^{\alpha} \Gamma_{\sigma \alpha}^{\mu}
$$

If we now replace $\sigma$ by $\beta, \nu$ by $\alpha$ and $\alpha$ by $\gamma$ in equation (25), we obtain:

$$
R_{\alpha \beta}=\Gamma_{\beta \alpha, \mu}^{\mu}+\Gamma_{\beta \alpha}^{\mu} \Gamma_{\mu \gamma}^{\gamma}-\Gamma_{\mu \alpha, \beta}^{\mu}-\Gamma_{\beta \gamma}^{\mu} \Gamma_{\mu \alpha}^{\gamma}
$$

where $\Gamma_{\alpha \beta, \sigma}^{\rho}$ denotes $\frac{\partial}{\partial x^{\sigma}} \Gamma^{\rho}{ }_{\alpha \beta}$. For $\mu, \alpha, \beta, \gamma=0,1,2,3$ and $\alpha=\beta=1$, equation (26) gives the Ricci tensor as:

$$
R_{11}=\Gamma_{11, \mu}^{\mu}+\Gamma_{11}^{\mu} \Gamma_{\mu \gamma}^{\gamma}-\Gamma_{\mu 1,1}^{\mu}-\Gamma_{1, \gamma}^{\mu} \Gamma_{\mu, 1}^{\gamma}
$$


The first term on the r.h.s of equation (27) gives:

$$
\begin{aligned}
& \Gamma_{11, \mu}^{\mu}=\Gamma_{11,0}^{0}+\Gamma_{11,1}^{1}+\Gamma_{11,2}^{2}+\Gamma_{11,3}^{3} \\
& =\frac{\partial}{\partial x}\left(\frac{-2 \kappa x}{1+\kappa r^{2}}\right)+\frac{\partial}{\partial y}\left(\frac{2 \kappa y}{1+\kappa r^{2}}\right)+\frac{\partial}{\partial z}\left(\frac{2 \kappa z}{1+\kappa r^{2}}\right) \\
& \quad+\frac{\partial}{\partial t}\left(\frac{R(t) R^{\prime}(t)}{c^{2}\left(1+\kappa r^{2}\right)^{2}}\right) \\
& =\frac{R(t) R^{\prime \prime}(t)+R^{\prime}(t)^{2}}{c^{2}\left(1+\kappa r^{2}\right)^{2}}+\frac{2 \kappa}{1+\kappa r^{2}}+\frac{4 \kappa^{2}\left(x^{2}-y^{2}-z^{2}\right)}{\left(1+\kappa r^{2}\right)^{2}}
\end{aligned}
$$

Similarly, other terms in equation (27) are calculated and found to be:

$$
\begin{gathered}
\Gamma_{11}^{\mu} \Gamma_{\mu \gamma}^{\gamma}=\frac{3 R^{\prime}(t)^{2}}{c^{2}\left(1+\kappa r^{2}\right)^{2}}+\frac{12 \kappa^{2}\left(x^{2}-y^{2}-z^{2}\right)}{\left(1+\kappa r^{2}\right)^{2}} \\
\Gamma^{\mu}{ }_{\mu 1,1}=\frac{-6 \kappa}{1+\kappa r^{2}}+\frac{12 \kappa^{2} r^{2}}{\left(1+\kappa r^{2}\right)^{2}} \\
\Gamma_{1, \gamma}^{\mu} \Gamma_{\mu, 1}^{\gamma}=\frac{2 R^{\prime}(t)^{2}}{c^{2}\left(1+\kappa r^{2}\right)^{2}}+\frac{2 \kappa^{2} x^{2}}{\left(1+\kappa r^{2}\right)^{2}}-\frac{8 \kappa^{2}\left(y^{2}+z^{2}\right)}{\left(1+\kappa r^{2}\right)^{2}}
\end{gathered}
$$

Using equations (28)-(31), we get the components of the Ricci tensor $R_{11}$ in equation (27) as

$$
R_{11}=\frac{R(t) R^{\prime \prime}(t)+2 R^{\prime}(t)^{2}+8 \kappa c^{2}}{c^{2}\left(1+\kappa r^{2}\right)^{2}}
$$

In the same manner it can be shown that the other non-vanishing values of the Ricci tensor are $R_{22}, R_{33}$ and $R_{00}$ such that

$$
R_{33}=R_{22}=R_{11}=\frac{R(t) R^{\prime \prime}(t)+2 R^{\prime}(t)^{2}+8 \kappa c^{2}}{c^{2}\left(1+\kappa r^{2}\right)^{2}}
$$

and

$$
R_{00}=\frac{-3 R^{\prime \prime}(t)}{R(t)}
$$

Raising the indices of $R_{\alpha \beta}$, equations (34) and (33) respectively gives:

$$
R_{0}^{0}=\frac{-3 R^{\prime \prime}(t)}{c^{2} R(t)}
$$

and

$$
R_{1}^{1}=R_{2}^{2}=R_{3}^{3}=-\frac{\left.8 \kappa c^{2}+R(t) R^{\prime \prime}(t)+2 R^{\prime}(t)^{2}\right)}{c^{2} R(t)}
$$


Therefore, the curvature scalar is:

$$
R=R_{\mu}^{\mu}=-3 \frac{\left(8 \kappa c^{2}+R(t) R^{\prime \prime}(t)+2 R^{\prime}(t)^{2}\right)}{c^{2} R(t)^{2}}
$$

Finally, the Einstein tensor $G^{\mu \nu}$ is:

$$
G^{\mu \nu}=R^{\mu \nu}-\frac{R g^{\mu \nu}}{2}
$$

The isotropy requires that the matter content in the universe must be at rest with respect to coordinates $x, y, z$ otherwise the direction of the velocity would break the isotropy. Furthermore, if the universe is assumed to be homogeneous, this matter content must be uniformly distributed everywhere. From the relativistic theory of fields and matter (Noether theorem of conservation), with every distribution of matter and fields, there is associated a symmetric tensor, i.e., the energy-momentum tensor, $T^{\mu \nu}$. The equivalence principle asserts that it is covariantly conserved as is also the Einstein tensor. The component $T^{\mu 0}$ for $\mu=0$ is the energy density while for $\mu=1,2,3$ are the momenta densities. The space components represents the current densities. However, in general, Noether theorem does not lead to a symmetric energy-momentum tensor $T^{\mu \nu}$, but there is always a modification of it which is symmetric i.e., $T^{\mu \nu}=T^{\nu \mu}$. The equivalence principle asserts that it is covariantly conserved as is also the Einstein tensor. The Einstein tensor is related to the stress-energy tensor of the matter content in the universe by the Einstein equation:

$$
G^{\mu \nu}=B T^{\mu \nu} ; \quad B=8 \pi G c^{-4}
$$

where $G$ is the gravitational constant. This tensor is uniform everywhere on a timeslice, i.e., must not depend on the coordinates $x, y, z$. This implies that the stress-energy tensor must take the form:

$$
T^{00}=\rho(t)
$$

and

$$
T^{11}=T^{22}=T^{33}=\frac{\left.\left(1+\kappa r^{2}\right)^{2}\right) p(t)}{R(t)^{2}},
$$

where $\rho(t)$ and $p(t)$ are the mass density and pressure (both functions of time), respectively. Equation (38) above contains two independent equations for $\mu=$ $\nu=0$ and $\mu=\nu=1,2,3$. Using equations (34) and (37) in equation (38), we can easily show that for $\mu=\nu=0$,

$$
G^{00}=R^{00}-\frac{R g^{00}}{2}=3 \frac{\left(4 \kappa c^{2}+R^{\prime}(t)^{2}\right)}{c^{4} R^{\prime}(t)^{2}}
$$


Using equations (40) and (42) in equation (38), we get Einstein equation:

$$
12 \kappa c^{2}+3 R^{\prime}(t)^{2}=B c^{4} R(t)^{2} \rho(t)
$$

Similarly, it can be shown that for $\mu=\nu=1,2,3$,

$$
G^{11}=G^{22}=G^{33}=-\frac{\left(4 \kappa c^{2}+2 R(t) R^{\prime \prime}(t)+R^{\prime}(t)^{2}\right)}{c^{2} R(t)^{4} /\left(1+\kappa r^{2}\right)}
$$

Substituting equations (41) and (44) into equation (38) yields:

$$
4 \kappa c^{2}+2 R(t) R^{\prime \prime}(t)+R^{\prime}(t)^{2}=-B c^{2} R(t)^{2} p(t)
$$

Equations (43) and (45) constitute our desired Einstein equations for describing the dynamics and evolution of the universe. We now proceed to develop a conservation law based on these equations.

\subsection{Conservation Law}

Differentiating equation (43) and multiplying the result by $R(t)$ we have:

$$
6 R(t) R^{\prime}(t) R^{\prime \prime}(t)=2 B c^{4} R(t)^{2} R^{\prime}(t) \rho(t)+B c^{4} R(t)^{3} \rho^{\prime}(t)
$$

Multiplying equation (45) by 3 and re-arranging it gives:

$$
6 R(t) R^{\prime \prime}(t)=-\left(12 \kappa c^{2}+3 R^{\prime}(t)^{2}\right)-3 B c^{2} R(t)^{2} p(t)
$$

Applying equation (43) in equation (47) and multiplying the result by $R^{\prime}(t)$ yields:

$$
6 R(t) R^{\prime}(t) R^{\prime \prime}(t)=-B c^{4} R^{\prime}(t) R(t)^{2} \rho(t)-3 B c^{2} R^{\prime}(t) R(t)^{2} p(t)
$$

Subtracting equation(48) from equation (46), we can write the result in the form:

$$
\frac{d}{d t}\left(c^{2} \rho(t) R(t)^{3}\right)=-p(t) \frac{d}{d t} R(t)^{3}
$$

So the rate of change of energy in the universe is the negative of the product of the pressure and the rate of change of the volume in the universe. The universe as we see it today seems to be matter-dominated. This matter seems to be uniformly distributed and is at rest w.r.t $t=$ constant hypersurfaces. This matter can be regarded as atoms of a gas which are at zero temperature hence has mass density $\rho(t)>0$ but zero pressure. From the above equation, it is 
clear that the conservation law for the stress energy tensor $T^{\mu \nu}$ for $p(t)=0$, says that the total mass contained in the universe remains constant, i.e., the mass density times the radius of the universe cubed is a constant say, $\alpha$ given by:

$$
\rho(t) R(t)^{3}=\alpha
$$

Equations (43) and (50) gives:

$$
12 \kappa c^{2}+3 R^{\prime}(t)^{2}=\frac{B c^{4} \alpha}{R(t)}
$$

implying that

$$
d t=\frac{d R}{\sqrt{\frac{B c^{2} \alpha}{3 R}-4 \kappa c^{2} R}}
$$

\section{Functional Dependency of Light Intensity on Redshift}

Now consider an emitted light ray from an astronomical object (e.g., a star) that starts at $r\left(t_{e}\right)$ and travels towards origin such that at time $t=0$, it reaches the origin i.e., $r\left(t_{o}\right)=0$.

$$
\frac{c^{2} \dot{t}^{2}-R^{2} \dot{r}^{2}}{\left(1+\kappa r^{2}\right)^{2}}=0
$$

or

$$
c \dot{t}=\frac{R(t) \dot{r}}{1+\kappa r^{2}}
$$

But $\dot{t}$ is positive while $\dot{r}$ is negative by our assumption, hence

$$
c d t=-\frac{R(t) d r}{1+\kappa r^{2}}
$$

Rearranging equation (53) and integrating, we have

$$
\int_{t_{e}}^{t_{o}} \frac{c}{R(t)} d t=-\int_{r\left(t_{e}\right.}^{r\left(t_{o}\right.} \frac{1}{1+\kappa r^{2}} d r
$$

Substituting equation (51) into equation (54) to change to integration over $R$, we have

$$
\int_{R\left(t_{e}\right)}^{R\left(t_{o}\right)} \frac{c d R}{\sqrt{R} \sqrt{\frac{B c^{4} \alpha}{3}-4 \kappa c^{2} R}}=-\int_{r\left(t_{e}\right.}^{r\left(t_{o}\right.} \frac{1}{1+\kappa r^{2}} d r
$$


But using the redshift equation

$$
R\left(t_{e}\right)=\frac{R\left(t_{o}\right)}{1+z}
$$

and noting that $r\left(t_{o}\right)=0$, we can show that for $\kappa=0,1,-1$, equation (55) can be written in a compact form as:

$$
\begin{gathered}
r\left(t_{e}\right)=\sqrt{12 R\left(t_{o}\right)}\left[\sqrt{B c^{2} \alpha(1+z)-12 \kappa R\left(t_{o}\right)}\right. \\
\left.-\sqrt{B c^{2} \alpha-12 \kappa R\left(t_{o}\right)}\right] / \\
\sqrt{B c^{2} \alpha-12 \kappa R\left(t_{o}\right)} \times \\
\sqrt{B c^{2} \alpha(1+z)-12 \kappa R\left(t_{o}\right)}+12 \kappa R\left(t_{o}\right)
\end{gathered}
$$

Defining

$$
a=B c^{2} \alpha-12 \kappa R\left(t_{o}\right) ; \quad b=B c^{2} \alpha(1+z)-12 \kappa R\left(t_{o}\right)
$$

and noting that $t_{e}$ itself depends on $z$, we consider $r\left(t_{e}\right)$ as a function $r(z)$ so that equation (57) can be re-written as:

$$
r(z)=\frac{\sqrt{12 R\left(t_{o}\right)}(\sqrt{b}-\sqrt{a})}{\sqrt{a b}+12 \kappa R\left(t_{o}\right)}
$$

To establish the relationship between the light intensity $I$ from an emitting astronomical object on the redshift, $z$ we consider a star emitting light at an absolute luminosity (power), $L$. Let the star be situated at the coordinate $r=0$. At time $t=t_{e}$, we consider the light emitted during an interval of time, $d t_{e}$. Suppose that at time $t=t_{o}$, an observer measures the brightness $I$ of that light which he receives at a redshift $z$, then his position of reception of the light is given by equation (59). This is because equation (59) is invariant on time reversal. This light that was emitted in the interval $\left.t_{e}, t_{e}+d t_{e}\right]$ will pass the observer in the time interval $\left[t_{o}, t_{o}+d t_{o}\right]$. During this process, what is conserved as the radiation passes through the universe is the number of photons. But each of this photons is red-shifted, hence its energy decreases by a factor $\frac{1}{1+z}$. Since this red-shifting is for all photons, this means that the energy that passes through the sphere of radius $r=r(z)$ during the interval $\left[t_{o}, t_{o}+d t_{o}\right]$ is the same as $\frac{1}{1+z}$ times the energy emitted during the interval $\left[t_{e}, t_{e}+d t_{e}\right]$. This implies that

$$
I=\frac{L d t_{e}}{(1+z) S_{r(z)}}
$$


where $S_{r(z)}$ denotes the surface area of the sphere of radius $r=r(z)$ at time $t=t_{o}$. Equation (53) can be rearranged and integrated from time $t_{e}$ of emission to time $t_{o}$ of observation and from the coordinate radius $r=0$ to $r=r(z)$ as:

$$
\int_{t_{e}}^{t_{o}} \frac{c}{R(t)} d t=\int_{o}^{r(z)} \frac{1}{1+\kappa r^{2}} d r
$$

Equation (61) can be written in terms of the time intervals as:

$$
\int_{t_{e}+d t_{e}}^{t_{o}+d t_{o}} \frac{c}{R(t)} d t=\int_{o}^{r(z)} \frac{1}{1+\kappa r^{2}} d r
$$

Applying the fundamental theorem of integral calculus to equation (62), we have:

$$
\frac{c d t_{o}}{R\left(t_{o}\right)}-\frac{c d t_{e}}{R\left(t_{e}\right)}+\int_{t_{e}}^{t_{o}} \frac{c}{R(t)} d t=\int_{o}^{r(z)} \frac{1}{1+\kappa r^{2}} d r
$$

If we substitute equation (61) into equation (63), we get:

$$
\frac{d t_{o}}{R\left(t_{o}\right)}=\frac{d t_{e}}{R\left(t_{e}\right)}
$$

which on application of equation (56) gives:

$$
\frac{d t_{e}}{d t_{o}}=\frac{R\left(t_{e}\right)}{R\left(t_{o}\right)}=\frac{1}{1+z}
$$

Substituting equation (65) into equation (60) and taking the surface area of the sphere $S_{r(z)}$ as $4 \pi r(z)^{2} R\left(t_{o}\right)^{2} /\left(1+\kappa r(z)^{2}\right)^{2}$ gives:

$$
I=\frac{L\left(1+\kappa r(z)^{2}\right)^{2}}{(1+z)^{2} 4 \pi r(z)^{2} R\left(t_{o}\right)^{2}}
$$

Using equation (59) in equation (66), we get:

$$
I=\frac{L\left[1+\kappa\left(\frac{\sqrt{12 R\left(t_{o}\right)}(\sqrt{b}-\sqrt{a})}{\sqrt{a b}+12 \kappa R\left(t_{o}\right)}\right)^{2}\right]^{2}}{(1+z)^{2} 4 \pi\left(\frac{\sqrt{12 R\left(t_{o}\right)}(\sqrt{b}-\sqrt{a})}{\sqrt{a b}+12 \kappa R\left(t_{o}\right)}\right)^{2} R\left(t_{o}\right)^{2}}
$$

Defining

$$
\beta_{1}=12 R\left(t_{o}\right)(\sqrt{b}-\sqrt{a}) ; \beta_{2}=\sqrt{a b}+12 \kappa R\left(t_{o}\right) ; r(z)=\frac{\beta_{1}}{\beta_{2}}
$$


and using in equation (67) yields:

$$
I=\frac{L\left[\left(\kappa \beta_{1}^{2}\right)^{2}+2 \kappa \beta_{1}^{2} \beta_{2}^{2}+\left(\beta_{2}^{2}\right)^{2}\right]}{4 \pi \beta_{1}^{2} R^{2}(1+z)^{2}}
$$

or

$$
I=\frac{L\left[\left(\kappa \beta_{1}\right)^{2}+2 \kappa \beta_{2}^{2}+\beta_{2}^{4} / \beta_{1}^{2}\right]}{4 \pi R^{2}(1+z)^{2}}
$$

Applying equation (68) to equation (70) gives:

$$
\begin{aligned}
I=L\left[\kappa ^ { 2 } \left(12 R\left(t_{o}\right)\right.\right. & \left.(\sqrt{b}-\sqrt{a})^{2}\right)+2 \kappa\left(\sqrt{a b}+12 \kappa R\left(t_{o}\right)\right)^{2} \\
& \left.+\frac{\left(\sqrt{a b}+12 \kappa R\left(t_{o}\right)\right)^{4}}{12 R\left(t_{o}\right)(\sqrt{b}-\sqrt{a})^{2}}\right] / 4 \pi R^{2}(1+z)^{2}
\end{aligned}
$$

Using equation (58) in equation (71), we get:

$$
\begin{aligned}
I=L\left[\kappa^{2}(\right. & 12 R\left(t_{o}\right)\left(\sqrt{B c^{2} \alpha(1+z)-12 \kappa R\left(t_{o}\right)}\right. \\
& \left.\left.-\sqrt{B c^{2} \alpha-12 \kappa R\left(t_{o}\right)}\right)^{2}\right)+2 \kappa\left(\sqrt{B c^{2} \alpha-12 \kappa R\left(t_{o}\right)} \times\right. \\
& \left.\sqrt{B c^{2} \alpha(1+z)-12 \kappa R\left(t_{o}\right)}+12 \kappa R\left(t_{o}\right)\right)^{2} \\
+ & \left\{\left(\left(\sqrt{B c^{2} \alpha-12 \kappa R\left(t_{o}\right)} \sqrt{B c^{2} \alpha(1+z)-12 \kappa R\left(t_{o}\right)}\right.\right.\right. \\
+ & \left.\left.\left.\left.-\sqrt{B c^{2} \alpha-12 \kappa R\left(t_{o}\right)}\right)^{2}\right)\right\}\right] / 4 \pi R^{2}(1+z)^{2}
\end{aligned}
$$

Making use of equations (39) and (50), equation (72) can be re-written as

$$
\begin{aligned}
I=L\left[\kappa ^ { 2 } \left(12 R\left(t_{o}\right)\right.\right. & \left(\sqrt{8 \pi G c^{-2} \rho\left(t_{o}\right) R\left(t_{o}\right)^{3}(1+z)-12 \kappa R\left(t_{o}\right)}\right. \\
& \left.\left.-\sqrt{8 \pi G c^{-2} \rho\left(t_{o}\right) R\left(t_{o}\right)^{3}-12 \kappa R\left(t_{o}\right)}\right)^{2}\right) \\
+ & 2 \kappa\left(\sqrt{8 \pi G c^{-2} 2\left(t_{o}\right) R\left(t_{o}\right)^{3}-12 \kappa R\left(t_{o}\right)} \times\right. \\
& \sqrt{8 \pi G c^{-2} \rho\left(t_{o}\right) R\left(t_{o}\right)^{3}(1+z)-12 \kappa R\left(t_{o}\right)}
\end{aligned}
$$




$$
\begin{gathered}
\left.+12 \kappa R\left(t_{o}\right)\right)^{2} \\
+\left\{\left[\sqrt{8 \pi G c^{-2} \rho\left(t_{o}\right) R\left(t_{o}\right)^{3}-12 \kappa R\left(t_{o}\right)} \times\right.\right. \\
\sqrt{8 \pi G c^{-2} \rho\left(t_{o}\right) R\left(t_{o}\right)^{3}(1+z)-12 \kappa R\left(t_{o}\right)} \\
\left.+12 \kappa R\left(t_{o}\right)\right]^{4} \\
/\left[1 2 R ( t _ { o } ) \left(\sqrt{8 \pi G c^{-2} \rho\left(t_{o}\right) R\left(t_{o}\right)^{3}(1+z)-12 \kappa R\left(t_{o}\right)}-\right.\right. \\
\left.\left.\left.\left.\sqrt{8 \pi G c^{-2} \rho\left(t_{o}\right) R(t-o)^{3}-12 \kappa R\left(t_{o}\right)}\right)^{2}\right]\right\}\right] \\
/ 4 \pi R^{2}(1+z)^{2}
\end{gathered}
$$

Equations (67)-(73) are alternative forms of equation (66). Equation (73) gives us the general relationship between $I$ and $z$ in terms of mass density and cosmic scale factor of the universe. Let us now consider our earlier equations in terms of these two parameters $\rho(t)$ and $R(t)$. Using equation (58), we can write $b$ in terms of $a$ as:

$$
b=a+B c^{2} \alpha z
$$

For small redshifts $z$ (nearby stars or galaxies), we can expand equation (74) as:

$$
\sqrt{b}=\sqrt{a}\left(1+\frac{1}{2} \frac{B c^{2} \alpha}{a} z-\frac{1}{8}\left(\frac{B c^{2} \alpha}{a}\right)^{2} z^{2}+---\right)
$$

Using equation (75) in equation (59) (considering the first two terms of the expansion), we get:

$$
r(z) \approx \sqrt{\frac{3 R\left(t_{o}\right) \alpha}{b+12 \kappa R\left(t_{o}\right)}} B c^{2} \alpha z
$$

Simplifying for small $z$ (for nearby stars or galaxies),

$$
r(z)=\sqrt{\frac{3 R\left(t_{o}\right)}{a}}
$$

This shows that $r(z)$ is proportional to $z$ for small $z$. We can re-write equation (77) by use of equations (39), (50) and (58) as:

$$
r(z)=\sqrt{\frac{3}{8 \pi G c^{-2} \rho\left(t_{o}\right) R^{2}\left(t_{o}\right)-12 \kappa}} z
$$


Similarly, it is easy to show for large values of $z$ that equation (59) becomes:

$$
r(z)=\sqrt{\frac{3}{2 \pi G c^{-2} \rho\left(t_{o}\right) R^{2}\left(t_{o}\right)-3 \kappa}}
$$

Substituting the result derived from equation $(78)$ that $r(z) \approx z ; \operatorname{kr}(z) \approx z$ into equation (66) for small $z$ gives

$$
I=\frac{L}{4 \pi R\left(t_{o}\right)^{2} z^{2}},
$$

so that $I$ is inversely proportional to the square of the redshift $z$. This result is in agreement with our classical expectation that the light intensity from nearby stars or galaxies (small $z$ ) obeys Newtonian physics in which the light intensity falls as the inverse of the square of the redshift $z$. Similarly, galaxies which are far away (large values of $z$ such that $1+z \approx z$ ), equation (66) gives:

$$
I=\frac{L\left(a+12 \kappa R\left(t_{o}\right)\right)^{2}}{48 \pi a R^{3}\left(t_{o}\right) z^{2}}=\frac{L \pi G^{2} c^{-4} \rho^{2}\left(t_{o}\right) R^{2}\left(t_{o}\right)}{3\left(2 \pi G c^{-2} \rho\left(t_{o}\right) R^{2}\left(t_{o}\right)-3 \kappa\right) z^{2}}
$$

Therefore, the light intensity from far off stars or galaxies also falls off as the inverse of the square of the redshift $z$.

\section{Discussion and Summary}

Direct distance measurements is only possible for nearby stars and galaxies even with advanced modern telescopes. In this paper, we have assumed that we are given huge and direct observational data from which the light intensity and the redshift have been measured from a certain class of astronomical objects that has constant absolute luminosity e.g., Type I supernova. Subject to the availability of such data that does not involve distance measurements and hence, no assumptions about the background geometry, we have derived a functional relationship between redshift and light intensity for nearby and distant astronomical objects. The functional relationship between these two astronomical quantities assumes an underlying Friedmann-Robertson-Walker metric that embodies the cosmological principle. The relation generates correct classical result for small redshifts of nearby astronomical objects as seen from equation (80). Indeed, for small values of $z$, the speed $v$ of a galaxy receding from us is proportional to the redshift $z$ by $v=c z$. Furthermore, the speed $v$ of a galaxy is also proportional to its distance $D$ from us through $v=H D$, where $H$ is the 
Hubble constant. Clearly, $D=\frac{c}{H} z$ hence, $D$ is proportional to $z$ as expected classically.

For distant astronomical bodies, the light intensity also falls as the inverse of the square of the redshift as given by equation (81). Equation (73) gives the general equation relating reshift $z$ and light intensity $I$ in terms of mass density $\rho\left(t_{o}\right)$ and cosmic scale factor $R\left(t_{o}\right)$ of the universe at observational time $t_{o}$. With appropriate choice of $\rho\left(t_{o}\right)$ and $R\left(t_{o}\right)$ and fixing of other parameter terms, one can constrain the relation between $I$ and $z$ for far and nearby astronomical bodies through computer simulations and consequently, determine the nature of the universe in question, i.e., whether it is flat, closed or open, depending on the values of $\kappa$. This will form the basis of our subsequent task. Therefore, from data that does not involve direct distance measurements, we have laid the basis for experimental work on verifying as to whether or not luminous matter is homogeneously distributed in accordance with the Friedmann universe.

\section{Acknowledgments}

I would like to thank University of Nairobi for financial support of this research work.

\section{References}

[1] B. V. Michal, Principles of Cosmology and Gravitation, Institute of Physics Publishing, England (1989).

[2] F. S. Labini, M. Montuori, L. Pietronero, Scale-invariance of Galaxy clustering, Physics Report, 293 (1998), 61-226. doi:10.1016/S0370-1573(97)00044-6.

[3] L. Pietronero, F. S. Labini, M. Montuori, The debate of galaxy correlations and its theoretical implications, Astrophysical Letters $\&$ Communications, 36, No-s: 1-6 (1997), 65-73.

[4] F. S. Labini, L. Pietronero, Multifractality as a link between luminosity and space distribution of visible matter, Astrophysical Journal, 469 (1996), 26-39. doi:10.1086/177754.

[5] M. Vergassola, B. Dubrulle, U. Frisch, A. Noullez, Burgers' equation, Devil's staircases and the mass distribution for large-scale structures, Astronomy \& Astrophysics, 289 (1994), 325-356.

[6] C. Meisner, K. S. Thorn, J. A. Wheeler, Gravitation, W.H. Freemann and Company, USA (1973).

[7] R. M. Wald, General Relatity, University of Chicago Press Ltd., London (1994).

[8] M. Potashov, S. Blinnikov, P. Baklanov, A. Dolgov, Direct distance measurements to SN 2009ip, Monthly Notices of the Royal Society, 000, (2012), 1-4. doi:10.1093/mnrasl/slt016. 
[9] G. Richard, Advancing the physics of cosmic distances: Conference summary, Proceedings IAU symbossium, 8, No. 289 (2012), 351-360. doi:10.1017/S1743921312021709.

[10] Y. Kazuhiro, Measuring cosmological parameters with the SDSS QSO spatial power spectrum, Monthly Notices of the Royal Society, 341 (2003), 1199-1204. doi:10.1046/j.13658711.2003.06477.x.

[11] M. Joyce, S.F. Labini, A. Gabrielli, M. Montuori, L. Pietronero, Basic properties of galaxy clustering in the light of recent results from the sloan digital sky survey, Astronomy 8 Astrophysics, 443 (2005), 11-16. doi:10.1051/0004-6361:20053658. 
